RU00-03-B

hep-th/0007053

\title{
RECOVERY OF THE SCHWARZSCHILD METRIC IN THEORIES WITH LOCALIZED GRAVITY BEYOND LINEAR ORDER
}

\author{
Ioannis Giannakis ${ }^{a}$ and Hai-cang $\operatorname{Ren}^{a, b} \dagger$ \\ (a) Physics Department, The Rockefeller University \\ 1230 York Avenue, New York, NY 10021-6399 \\ ${ }^{(b)}$ Department of Natural Science, Baruch College of CUNY \\ New York, NY 10010
}

\begin{abstract}
We solve the Einstein equations in the Randall-Sundrum framework with a static, spherically symmetric matter distribution on the physical brane and obtain an approximate expression for the gravitational field outside the source to the second order in the gravitational coupling. This expression when confined on the physical brane coincides with the standard form of the Schwarzschild metric. Therefore, the RandallSundrum scenario is consistent with the Mercury precession and other tests of General Relativity.
\end{abstract}

$\dagger$ e-mail: giannak@theory.rockefeller.edu,ren@theory.rockefeller.edu, 


\section{Introduction.}

Motivated by the hierarchy problems of the fundamental interactions, Randall and Sundrum have proposed an interesting scenario of extra non-compact dimensions in which four-dimensional gravity emerges as a low energy effective theory [1]. This novel proposal consists of two basic components. The first one is based on the assumption that ordinary matter and its gauge interactions are confined within a four dimensional hypersurface, referred to subsequently as the physical brane, embedded in a five-dimensional space [2]. The second ingredient involves the realization that a bound state of a five-dimensional graviton exists and is localized near the physical brane. Consequently, gravitational interactions at large distances on the physical brane are dominated by the bound state, and thus gravity appears effectively as four-dimensional. Modifications of this scenario include supersymmetric generalizations [3] and models in which gravity is mediated with metastable graviton bound states [4]. Different issues concerning these models have been discussed in [5]. The Randall-Sundrum model consists of a three-brane embedded in a five-dimensional space which is asymptotically anti-de Sitter. A generic form of the metric in this space is

$$
d s^{2}=e^{-2 \kappa|y|} \bar{g}_{\mu \nu} d x^{\mu} d x^{\nu}+d y^{2}
$$

the four-dimensional metric $\bar{g}_{\mu \nu}$ being asymptotically flat. The asymptotic metric with $\bar{g}_{\mu \nu}=\eta_{\mu \nu}$ satisfies the vacuum Einstein equations

$$
R_{m n}-\frac{1}{2} R g_{m n}-\Lambda g_{m n}=0
$$

with the cosmological constant $\Lambda=-6 \kappa^{2}$. Here and throughout the paper, we adopt the convention that the Greek indices take values $0-3$ and the Latin indices $0-4$. The striking feature of this scenario is the existence of a massless graviton bound state which corresponds to the metric fluctuations $h_{\mu \nu}=g_{\mu \nu}-\eta_{\mu \nu}$, constant in $y$ but nevertheless normalizable because of the conformal factor $e^{-2 \kappa|y|}$. In the absence of nonzero modes (i.e., for a $y$ independent metric $\bar{g}_{\mu \nu}$ ) the five-dimensional Einstein equations with negative cosmological constant reduce to the four-dimensional Einstein equations in vacuum without a cosmological constant. Thus the Randall-Sundrum scenario can be extended, provided that the metric $\bar{g}_{\mu \nu}$ is Ricci flat, i.e., corresponds to any vacuum solution of General Relativity. This can be seen as follows: The Einstein equations can be rewritten as

$$
R_{\mu \nu}-4 \kappa^{2} g_{\mu \nu}=0, \quad R_{4 \mu}=0, \quad R_{44}-4 \kappa^{2}=0
$$

with $g_{\mu \nu}=e^{-2 \kappa|y|} \bar{g}_{\mu \nu}$. If we express the five dimensional Ricci tensor in terms of the four dimenional metric $\bar{g}_{\mu \nu}$, we find

$$
R_{\mu \nu}=\bar{R}_{\mu \nu}+4 \kappa^{2} g_{\mu \nu}
$$


for a $y$ independent metric $\bar{g}_{\mu \nu}$. Consequently, $\bar{g}_{\mu \nu}$ obeys $\bar{R}_{\mu \nu}=0$. An example is the Schwarzschild solution [6]

$$
d s^{2}=e^{-2 \kappa|y|}\left[-\left(1-\frac{2 G M}{r}\right) d t^{2}+\frac{1}{1-\frac{2 G M}{r}} d r^{2}+r^{2} d \Omega^{2}\right]+d y^{2}
$$

the source of which is a mass line extending infinitely in the $y$-direction. Another example is the pp-wave solution which describes gravitational waves propagating on the brane [7]. In the context of a physical situation-a spherically symmetric mass distribution located on the physical brane-nonzero modes are expected to contribute to the dynamics. The subsequent $y$-dependence of the metric introduces difficulties in solving the Einstein equations exactly. How these modes localize the matter induced curvature near the brane yet maintain on the brane the asymptotic agreement between the solutions of the five-dimensional Einstein equations and the four-dimensional ones has only been shown explicitly for linear gravity. In this paper, we shall extend such an analysis to the first order of nonlinearity ( second order in the gravitational coupling ) for the static axially symmetric metric produced by spherically symmetric matter that is localized on the brane.

Similar to the case of linearized gravity, the solution to the Einstein equations can be formulated as a solution free from any metric singularity far away from the source when it is expressed in terms of coordinates that are straight with respect to the horizon. We shall eventually transform the solution to a new coordinate system-natural coordinates of the brane-by performing a gauge transformation that is determined by the Israel matching condition [8] ( Discussion on these issues concerning linearized gravity appear in [9]). We shall work in the weak field approximation and in a region outside the material source where the Israel matching condition reduces to the Neumann boundary condition. *

Our solution, in contrast to the black-string solution [6], will explore both the far away region on the brane and the $A d S_{5}$ horizon. The arbitrary parameters of the solution are determined by the Newtonian limit on the brane.

The apparent gauge dependence of the solution is the consequence of the incompleteness of the Randall-Sundrum scenario. Indeed, as it was pointed out in [10], all material particles fall freely towards the $A d S_{5}$ horizon. Additional non-gravitational mechanisms are required to stabilize the brane and confine all material particles within the brane. In such models, possibly derived from string theory, the geodesic equation of material particles would include extra contributions. But it is still plausible that the Israel matching

* The physical brane contributes to the energy-momentum tensor as well. In the absence of matter, the metric $d s^{2}=e^{-2 \kappa|y|} \eta_{\mu \nu} d x^{\mu} d x^{\nu}+d y^{2}$ implies that $T_{\mu \nu}=m \eta_{\mu \nu} \delta(y), T_{4 \mu}=$ $T_{44}=0$, where $\mathrm{m}$ is a constant. Its natural extention in the presence of matter is $T_{\mu \nu}=m \bar{g}_{\mu \nu} \delta(y), T_{4 \mu}=T_{44}=0$. The linearity of Einstein's equation with respect to the second order derivatives leads to Neumann boundary condition. The same condition also guarantees the conservation of $T_{\mu \nu}$. 
condition selects a gauge in which the motion of material particles within the brane is given by the simple four-dimensional geodesic equation

$$
\frac{d^{2} x^{\mu}}{d s^{2}}+\Gamma_{\rho \lambda}^{\mu} \frac{d x^{\rho}}{d s} \frac{d x^{\lambda}}{d s}=0
$$

In order to describe the real world, the Randall-Sundrum scenario has to satisfy all the existing tests of General Relativity. Within the linearized gravity, it has been shown that the Newtonian limit as well as the spatial part of the Schwartzschild metric can be recovered on the physical brane for $\kappa r>>1$ with corrections from the nonzero graviton modes to the $1 / r$ terms of the order $O\left(1 / \kappa^{2} r^{3}\right)$ [1], [11], [10], [12]. Thus the scenario implies a deflection angle of light within the experimental bounds. For the precession angle of Mercury, the observation accuracy is up to the first order of nonlinearity of the metric component $g_{00}$. Indeed, for the weak field expansion of the static spherical metric

$$
d s^{2}=-\left(1-\frac{2 G M}{r}-\gamma \frac{G^{2} M^{2}}{r^{2}}+\ldots\right) d t^{2}+\left(1+\frac{2 G M}{r}+\ldots\right) d r^{2}+r^{2} d \Omega^{2}
$$

the precession angle measured in radians per revolution is given by [13]

$$
\delta=\frac{\pi G M}{L}(6+\gamma)
$$

where $L$ is the semilatus rectum of the Mercury orbit and $\gamma=0$ for the General Relativity. It is therefore imperative that the large $r$ behavior of $g_{00}$ on the brane in the RandallSundrum scenario is examined beyond linearized approximation. Our perturbative solution reproduces the General Relativity result $\gamma=0$. Thus the Randall-Sundrum scenario is consistent with all experimental tests of General Relativity.

For the sake of simplicity, we shall assume that the physical brane is located at $y=0$. In the next section we shall derive the Einstein equations for a static axially symmetric five dimensional metric, investigate the gauge symmetries-coordinate trasformations that respect the particular form of the metric-and discuss the appropriate boundary conditions. In section three we shall obtain an exact solution to the linearized equations, a solution which is free of curvature singularities and describes the gravitational field produced by a spherically symmetric mass distribution located on the brane. In section four, we will derive an approximate expression of the metric to second order in the gravitational coupling (first order in non-linearity) which is valid in a region far from the mass source. This expression when confined on the physical brane is in agreement with the standard four dimensional Schwarzschild metric and thus reproduces the correct precession angle of Mercury. Finally we will discuss the physical implications of our results. 
Schwarzschild metric beyond linear order ...

\section{Einstein Equations, Symmetries and Boundary Conditions.}

The most general axially symmetric and static metric in $D=4+1$ has the following form

$$
d s^{2}=e^{-2 \kappa|y|}\left(-e^{a} d t^{2}+e^{b} d r^{2}+e^{c} r^{2} d \Omega^{2}\right)+d y^{2},
$$

where $d \Omega^{2}=d \theta^{2}+\sin ^{2} \theta d \phi^{2}$ is the solid angle on $S^{2}$ and $a, b$ and $c$ are functions of $r$ and $y$. Substituting the metric (9) into (3) we obtain the following equations

$$
\begin{aligned}
& -a^{\prime \prime}-\frac{2}{r} a^{\prime}+\frac{1}{2} a^{\prime}\left(-a^{\prime}+b^{\prime}-2 c^{\prime}\right)-e^{-2 \kappa y+b}\left[\ddot{a}-5 \kappa \dot{a}-\kappa \dot{b}-2 \kappa \dot{c}+\frac{1}{2} \dot{a}(\dot{a}+\dot{b}+2 \dot{c})\right]=0 \\
& a^{\prime \prime}+2 c^{\prime \prime}-\frac{2}{r} b^{\prime}+\frac{4}{r} c^{\prime}+\frac{1}{2} a^{\prime}\left(a^{\prime}-b^{\prime}\right)-c^{\prime}\left(b^{\prime}-c^{\prime}\right) \\
& +e^{-2 \kappa y+b}\left[\ddot{b}-5 \kappa \dot{b}-\kappa \dot{a}-2 \kappa \dot{c}+\frac{1}{2} \dot{b}(\dot{a}+\dot{b}+2 \dot{c})\right]=0 \\
& c^{\prime \prime}+\frac{4}{r} c^{\prime}+\frac{a^{\prime}-b^{\prime}}{r}+c^{2}+\frac{1}{2}\left(a^{\prime}-b^{\prime}\right) c^{\prime} \\
& +e^{-2 \kappa y+b}\left[\ddot{c}-\kappa(\dot{a}+\dot{b})-6 \kappa \dot{c}+\frac{1}{2} \dot{c}(\dot{a}+\dot{b}+2 \dot{c})\right]-2 \frac{e^{b-c}-1}{r^{2}}=0 \\
& \ddot{a}+\ddot{b}+2 \ddot{c}-2 \kappa(\dot{a}+\dot{b}+2 \dot{c})+\frac{1}{2}\left(\dot{a}^{2}+\dot{b}^{2}+2 \dot{c}^{2}\right)=0 \\
& \dot{a}^{\prime}+2 \dot{c}^{\prime}-\frac{2}{r}(\dot{b}-\dot{c})+\frac{1}{2} a^{\prime}(\dot{a}-\dot{b})-c^{\prime}(\dot{b}-\dot{c})=0
\end{aligned}
$$

where the prime denotes a partial derivative with respect to $r$ and the dot denotes a partial derivative with respect to $y$. These equations apply to the positive side of the brane, i.e., $y>0$, the corresponding equations to the negative side of the brane, $y<0$, is obtained by switching the sign of $\kappa$.

For $y$-independent $a, b$ and $c$, equations (13) are satisfied automatically and equations (10), (11) and (12) become those of a static, spherically symmetric and Ricci flat metric albeit not asymptotically anti-de Sitter.

We shall discuss the gauge symmetries of these equations, more specifically the coordinate transformations that respect the axially symmetric, static form of the metric. Let's perform a coordinate transformation (gauge transformation) generated by $v, u$, functions of $r$ and $y$ such that $r \mapsto r+v(r, y)$ and $y \mapsto y+u(r, y)$ and demand that this particular coordinate transformation respects the form of the five dimensional metric (9). We find that the functions $v, u$ obey the following relations

$$
\begin{aligned}
& 2 \dot{v}+\dot{v}^{2}+e^{-2 \kappa y-2 \kappa v+b(r+u, y+v)} \dot{u}^{2}=0 \\
& v^{\prime}(1+\dot{v})+e^{-2 \kappa y-2 \kappa v+b(r+u, y+v)} \dot{u}\left(1+u^{\prime}\right)=0
\end{aligned}
$$


while the components of the metric transform under these residual coordinate transformations as follows

$$
\begin{aligned}
& a(r, y) \mapsto a(r+u, r+v)-2 \kappa v \\
& b(r, y) \mapsto b(r+u, y+v)-2 \kappa v+\ln \left[\left(1+u^{\prime}\right)^{2}+e^{2 \kappa y+2 \kappa v-b(r+u, y+v)} v^{\prime 2}\right] \\
& c(r, y) \mapsto c(r+u, y+v)-2 \kappa v+2 \ln \left(1+\frac{u}{r}\right) .
\end{aligned}
$$

In what follows, we shall construct the solutions to the Einstein equations in two different coordinate systems, or equivalently, in two different gauges [11], [14]. The solution in one coordinate system-the one in which the coordinates are straight with respect to the horizon-is free from metric singularities far away from the source. We shall denote this solution by $a(r, y), b(r, y)$ and $c(r, y)$ without superscripts and we shall impose the following conditions

$$
\lim _{|y|, r \rightarrow \infty} a=\lim _{|y|, r \rightarrow \infty} b=\lim _{|y|, r \rightarrow \infty} c=0
$$

independent of the order of the limits. The other set of coordinates are the natural coordinates of the brane. In this coordinate system the solution is given by the right hand side of (15) with the parameters $u$ and $v$ chosen appropriately to satisfy the Israel matching condition. We will denote the solution in this gauge by $a^{P}(r, y), b^{P}(r, y)$ and $c^{P}(r, y)$, where the superscript $P$ means "physical". Outside the material source, they satisfy the Neumann boundary condition, $\dot{a}^{P}(r, 0)=\dot{b}^{P}(r, 0)=\dot{c}^{P}(r, 0)=0$. In the subsequent sections, we shall use the following notation

$$
\begin{aligned}
& a^{P}(r, y)=a(r, y)+\delta a(r, y) \\
& b^{P}(r, y)=b(r, y)+\delta b(r, y) \\
& c^{P}(r, y)=c(r, y)+\delta c(r, y)
\end{aligned}
$$

where $\delta a, \delta b$ and $\delta c$ are not necessarily infinitesimal.

The Newtonian limit is specified by the asymptotic behavior of $g_{00}$ on the brane for large $r$, i.e.

$$
a^{P}(r, y)=1-\frac{2 G M}{r}-\cdots
$$

with $G$ being the four-dimensional gravitational constant and $M$ the total mass of the sourse. For a weak gravitational field, $G M<<r$, we have the following perturbative expansions for the solution that is free of metric singularities

$$
\begin{aligned}
& a(r, y)=a_{0}(r, y)+a_{1}(r, y)+\cdots, \quad b(r, y)=b_{0}(r, y)+b_{1}(r, y)+\cdots \\
& c(r, y)=c_{0}(r, y)+c_{1}(r, y)+\cdots
\end{aligned},
$$

and for the physical solution

$$
\begin{aligned}
& a^{P}(r, y)=a_{0}^{P}(r, y)+a_{1}^{P}(r, y)+\cdots, \quad b^{P}(r, y)=b_{0}^{P}(r, y)+b_{1}^{P}(r, y)+\cdots \\
& c^{P}(r, y)=c_{0}^{P}(r, y)+c_{1}^{P}(r, y)+\cdots
\end{aligned}
$$


Similarly we can expand the parameters of transformation

$$
\begin{aligned}
& v(r, y)=v_{0}(r, y)+v_{1}(r, y)+\cdots \\
& u(r, y)=u_{0}(r, y)+u_{1}(r, y)+\cdots
\end{aligned}
$$

All quantities with the subscript " 0 " are of the order $G M$ and those with the subscript "1" are of the order $G^{2} M^{2}$. In the next section we shall derive an exact solution to the first order in the gravitational coupling and we will discuss its physical significance.

\section{Linearized Solution.}

For a weak gravitational field, we obtain the linearized Einstein equations, i.e.

$$
\begin{aligned}
& -a_{0}^{\prime \prime}-\frac{2}{r} a_{0}^{\prime}-e^{-2 \kappa|y|}\left[\ddot{a}_{0}-5 \kappa \dot{a}_{0}-\kappa \dot{b}_{0}-2 \kappa \dot{c}_{0}\right]=0 \\
& a_{0}^{\prime \prime}+2 c_{0}^{\prime \prime}-\frac{2}{r} b_{0}^{\prime}+\frac{4}{r} c_{0}^{\prime}+e^{-2 \kappa y}\left[\ddot{b}_{0}-5 \kappa \dot{b}_{0}-\kappa \dot{a}_{0}-2 \kappa \dot{c}_{0}\right]=0 \\
& c_{0}^{\prime \prime}+\frac{4}{r} c_{0}^{\prime}+\frac{a_{0}^{\prime}-b_{0}^{\prime}}{r}+e^{-2 \kappa y}\left[\ddot{c}_{0}-\kappa\left(\dot{a}_{0}+\dot{b}_{0}\right)-6 \kappa \dot{c}_{0}\right]-\frac{2}{r^{2}}\left(b_{0}-c_{0}\right)=0, \\
& \ddot{a}_{0}+\ddot{b}_{0}+2 \ddot{c}_{0}-2 \kappa\left(\dot{a}_{0}+\dot{b}_{0}+2 \dot{c}_{0}\right)=0 \\
& \dot{a}_{0}^{\prime}+2 \dot{c}_{0}^{\prime}-\frac{2}{r}\left(\dot{b}_{0}-\dot{c}_{0}\right)=0 .
\end{aligned}
$$

Initially, we shall seek solutions that satisfy equation (16). The fourth of these equations is particularly simple, and the only solution consistent with the condition (16) is

$$
a_{0}+b_{0}+2 c_{0}=0
$$

Similarly, the last of equations (22) can be integrated to yield

$$
a_{0}^{\prime}+2 c_{0}^{\prime}-\frac{2}{r}\left(b_{0}-c_{0}\right)=\phi(r)
$$

where $\phi$ is an arbitrary function of $r$. The boundary condition (16) then demands that

$$
a_{0}^{\prime}+2 c_{0}^{\prime}-\frac{2}{r}\left(b_{0}-c_{0}\right)=0
$$

Note that the linearized Schwarzschild solution [6] satisfies (24) but not (25) . Substituting (23) into the first of equations (22) and using the Green's function found in [15], we obtain

$$
a_{0}(r, y)=-\frac{8 G M}{3 \pi \kappa} e^{2 \kappa y} \int_{0}^{\infty} d p p j_{0}(p r) \frac{K_{2}\left(\hat{p} e^{\kappa y}\right)}{K_{1}(\hat{p})}
$$


where $\hat{p}=p / \kappa . K_{\nu}(z)$ is the modified Bessel function of the second kind and $j_{0}(x)$ is the spherical Bessel function. The choice of the coefficient of the equation (26) is dictated by the requirement that it reproduces the correct Newtonian limit. Combining (23) and (25), we obtain a first order differential equation for $b$

$$
b_{0}^{\prime}+\frac{3}{r} b_{0}+\frac{a_{0}}{r}=0,
$$

the solution of which reads

$$
b_{0}(r, y)=\frac{8 G M}{3 \pi \kappa} e^{2 \kappa y} \int_{0}^{\infty} d p p \frac{j_{1}(p r)}{p r} \frac{K_{2}\left(\hat{p} e^{\kappa y}\right)}{K_{1}(\hat{p})} .
$$

It follows then from $(23)$ then that

$$
c_{0}(r, y)=\frac{8 G M}{3 \pi \kappa} e^{2 \kappa y} \int_{0}^{\infty} d p p \frac{1}{2}\left[j_{0}(p r)-\frac{j_{1}(p r)}{p r}\right] \frac{K_{2}\left(\hat{p} e^{\kappa y}\right)}{K_{1}(\hat{p})} .
$$

It is straightforward to verify that the solutions (26), (28) and (29) satisfy the two of the remaining equations (22) which we have not used.

If either $r$ or $\zeta \equiv \frac{1}{\kappa} e^{\kappa y}$ becomes large, i.e, $\kappa r>>1$ or $\kappa z>>1$, the integrals (26), (28) and (29) are dominated by the region where $\hat{p}<<1$. The modified Bessel function in the denominator, $K_{1}(\hat{p}) \simeq \frac{1}{\hat{p}}$, and the integrals can be carried out explicitly. We find that

$$
\begin{aligned}
& a_{0}(r, \zeta)=-\frac{4 G M}{3} \frac{2 r^{2}+3 \zeta^{2}}{\left(r^{2}+\zeta^{2}\right)^{\frac{3}{2}}}=-\frac{4 G M}{3 s}\left(2+\cos ^{2} \theta\right) \\
& b_{0}(r, \zeta)=\frac{4 G M}{3} \frac{1}{\sqrt{r^{2}+\zeta^{2}}}=\frac{4 G M}{3 s} \\
& c_{0}(r, \zeta)=\frac{2 G M}{3} \frac{r^{2}+2 \zeta^{2}}{\left(r^{2}+\zeta^{2}\right)^{\frac{3}{2}}}=\frac{2 G M}{3 s}\left(1+\cos ^{2} \theta\right)
\end{aligned}
$$

where we have introduced four-dimensional spherical coordinates $s$ and $\theta$ via $r=s \sin \theta$ and $\zeta=s \cos \theta$. In terms of $s$, and $\theta$ the physical brane corresponds to $\cos \theta=\frac{1}{\kappa r}$. We are primarily interested in the region with $\cos \theta<<1$. The result for $a_{0}(r, y)$ was obtained in [15]. Here we would like to emphasize that the expressions on the right hand sides of (30) approximate the entire region of $\kappa s>>1$. So it does interpolate the large $r$ region on the brane and the large $z$ region near the horizon. Furthermore, as is shown in the appendix, systematic inclusion of the higher order terms of $K_{1}(\hat{p})$ will lend us a large $s$ expansion of the type

$$
\frac{1}{s} \sum_{n=0}^{\infty} \frac{P_{n}(\theta ; \ln \kappa s)}{s^{2 n}}
$$

where $P_{n}(\theta, \ln \kappa s)$ is a $n$-th order polynomial in $\ln \kappa s$ with $\theta$-dependent coefficients. 
It is straightforward to check that while $a_{0}$ satisfies the Neumann boundary condition, $b_{0}$ and $c_{0}$ fail to do so. In order to transform the solution to the physical one we need to determine the parameters $v_{0}$ and $u_{0}$. Equations (14) read to first order as

$$
\dot{v}_{0}=0, \quad v_{0}^{\prime}+e^{-2 \kappa y} \dot{u}_{0}=0
$$

while the transformations of the components of the metric become

$$
\delta a_{0}=-2 \kappa v_{0}, \quad \delta b_{0}=-2 \kappa v_{0}+2 u_{0}^{\prime}, \quad \delta c_{0}=-2 \kappa v_{0}+2 \frac{u_{0}}{r} .
$$

We can easily verify that the Einstein equations (22) remain invariant under the transformations (32). Thus, the physical solution will be given by

$$
a_{0}^{P}=a_{0}+\delta a_{0}, \quad b_{0}^{P}=b_{0}+\delta b_{0}, \quad c_{0}^{P}=c_{0}+\delta c_{0} .
$$

We impose Neumann boundary condition on $b_{0}^{P}$ on the brane $\left.\dot{b}_{0}^{P}\right|_{y=0}=0$. Under a coordinate transformation generated by $v_{0}, u_{0}$, we find that

$$
\left.\dot{b}_{0}^{P}\right|_{y=0}=\frac{-4 G M}{\kappa r^{3}}+2 u_{0}^{\prime}=\frac{-4 G M}{\kappa r^{3}}-2 v_{0}^{\prime \prime}=0
$$

Thus,

$$
v_{0}(r)=-\frac{G M}{3 \kappa r}, \quad u_{0}(r, y)=-\frac{G M}{6 \kappa^{2} r^{2}} e^{2 \kappa y}+\chi(r)
$$

where $\chi$ is an arbitrary function of $r$ and is chosen $\chi=-\frac{2 G M}{3}$ in order to recover the standard Schwarzschild metric on the physical brane. The physical solution to first order takes then the form

$$
\begin{aligned}
& a_{0}^{P}=-\frac{8 G M}{3 \pi \kappa} e^{2 \kappa y} \int_{0}^{\infty} d p p j_{0}(p r) \frac{K_{2}\left(\hat{p} e^{\kappa y}\right)}{K_{1}(\hat{p})}+\frac{2 G M}{3 r} \\
& b_{0}^{P}=\frac{8 G M}{3 \pi \kappa} e^{2 \kappa y} \int_{0}^{\infty} d p p \frac{j_{1}(p r)}{p r} \frac{K_{2}\left(\hat{p} e^{\kappa y}\right)}{K_{1}(\hat{p})}+\frac{2 G M}{3 r}+\frac{2 G M}{3 \kappa^{2} r^{3}} e^{2 \kappa y} \\
& c_{0}^{P}=\frac{8 G M}{3 \pi \kappa} e^{2 \kappa y} \int_{0}^{\infty} d p p \frac{1}{2}\left[j_{0}(p r)-\frac{j_{1}(p r)}{p r}\right] \frac{K_{2}\left(\hat{p} e^{\kappa y}\right)}{K_{1}(\hat{p})}-\frac{2 G M}{3 r}-\frac{G M}{3 \kappa^{2} r^{3}} e^{2 \kappa y}
\end{aligned}
$$

It is straightforward to verify that $h_{0}^{P}=a_{0}^{P}+b_{0}^{P}+2 c_{0}^{P}=0$. Using equations (30), the metric on the physical brane for $r>>1 / \kappa$ becomes

$$
d s^{2}=-\left(1-\frac{2 G M}{r}+\cdots\right) d t^{2}+\left(1+\frac{2 G M}{r}+\cdots\right) d r^{2}+r^{2} d \Omega^{2}
$$

thus reproducing the standard form of the Schwarzschild metric. The dots in equation (38) represent terms of order $O\left(\frac{G^{2} M^{2}}{r^{2}}\right)$ and higher. 
Schwarzschild metric beyond linear order ...

\section{Second Order Approximate Solution.}

In this section, we will go beyond the first order solution (linearized) and find an approximate solution to second order in the gravitational coupling of the Einstein equations. Inserting the ansatz (20) to equations (10)-(13) and keeping terms to second order, we find

$$
\begin{gathered}
-a_{1}^{\prime \prime}-\frac{2}{r} a_{1}^{\prime}+\frac{1}{2} a_{0}^{\prime}\left(-a_{0}^{\prime}+b_{0}^{\prime}-2 c_{0}^{\prime}\right)-e^{-2 \kappa y}\left[\ddot{a}_{1}-5 \kappa \dot{a}_{1}-\kappa \dot{b}_{1}-2 \kappa \dot{c}_{1}+\frac{1}{2} \dot{a}_{0}\left(\dot{a}_{0}+\dot{b}_{0}+2 \dot{c}_{0}\right)\right. \\
\left.+b_{0}\left(\ddot{a}_{0}-5 \kappa \dot{a}_{0}-\kappa \dot{b}_{0}-2 \kappa \dot{c}_{0}\right)\right]=0 \\
\ddot{a}_{1}+\ddot{b}_{1}+2 \ddot{c}_{1}-2 \kappa\left(\dot{a}_{1}+\dot{b}_{1}+2 \dot{c}_{1}\right)+\frac{1}{2}\left(\dot{a}_{0}^{2}+\dot{b}_{0}^{2}+2 \dot{c}_{0}^{2}\right)=0 \\
\dot{a}_{1}^{\prime}+2 \dot{c}_{1}^{\prime}-\frac{2}{r}\left(\dot{b}_{1}-\dot{c}_{1}\right)+\frac{1}{2} a_{0}^{\prime}\left(\dot{a}_{0}-\dot{b}_{0}\right)-c_{0}^{\prime}\left(\dot{b}_{0}-\dot{c}_{0}\right)=0
\end{gathered}
$$

If we introduce $h_{1}=a_{1}+b_{1}+2 c_{1}$ it follows then from (40) and taking into account equation (30) that

$$
\dot{h}_{1}=\frac{\kappa G^{2} M^{2} \zeta^{2}}{\left(r^{2}+\zeta^{2}\right)^{2}}\left[\frac{11}{18}+\frac{5}{9} \frac{\zeta^{2}}{\left(r^{2}+\zeta^{2}\right)}+\frac{3}{2} \frac{\zeta^{4}}{\left(r^{2}+\zeta^{2}\right)^{2}}\right] .
$$

Consequently, integration over $y$ yields

$$
h_{1}=-\frac{G^{2} M^{2}}{\left(r^{2}+\zeta^{2}\right)}\left[\frac{25}{36}+\frac{7}{18} \frac{\zeta^{2}}{\left(r^{2}+\zeta^{2}\right)}+\frac{1}{4} \frac{\zeta^{4}}{\left(r^{2}+\zeta^{2}\right)^{2}}\right] \text {. }
$$

Taking into account (23), equation (40) becomes

$$
a_{1}^{\prime \prime}+\frac{2}{r} a_{1}^{\prime}+e^{-2 \kappa y}\left[\ddot{a}_{1}-4 \kappa \dot{a}_{1}\right]=a_{0}^{\prime} b_{0}^{\prime}-e^{-2 \kappa y} b_{0}^{\prime}\left(\ddot{a}_{0}-4 \kappa \dot{a}_{0}\right)+e^{-2 \kappa y} \kappa \dot{h}_{1}
$$

with the right hand side completely known. We proceed to introduce spherical coordinates $s, \theta$ such that $r=s \cos \theta, z=\frac{1}{\kappa} e^{\kappa y}=s \sin \theta$. In terms of these new variables, equation (40) takes the following form

$$
\left[\frac{1}{s^{3}} \frac{\partial}{\partial s}\left(s^{3} \frac{\partial}{\partial s}\right)+\frac{1}{s^{2} \sin ^{2} \theta} \frac{\partial}{\partial \theta}\left(\sin ^{2} \theta \frac{\partial}{\partial \theta}\right)-\frac{3}{s} \frac{\partial}{\partial s}+\frac{3}{s^{2}} \tan \theta \frac{\partial}{\partial \theta}\right] a_{1}(s, \theta)=f_{1}+f_{2}+f_{3}
$$

where

$$
\begin{aligned}
& f_{1}=a_{0}^{\prime} b_{0}^{\prime}=-\frac{16 G^{2} M^{2}}{9} \frac{\left(2 r^{2}+5 \zeta^{2}\right) r^{2}}{\left(r^{2}+\zeta^{2}\right)^{4}}=-\frac{16 G^{2} M^{2}}{9} \frac{2+\cos ^{2} \theta-3 \cos ^{4} \theta}{s^{4}} \\
& f_{2}=-e^{-2 \kappa y} b_{0}^{\prime}\left(\ddot{a}_{0}-4 \kappa \dot{a}_{0}\right)=\frac{16 G^{2} M^{2}}{3} \frac{5 \zeta^{4}}{\left(r^{2}+\zeta^{2}\right)^{4}}=\frac{16 G^{2} M^{2}}{3} \frac{5 \cos ^{4} \theta}{s^{4}} \\
& f_{3}=e^{-2 \kappa y} \kappa\left(\dot{a}_{1}+\dot{b}_{1}+2 \dot{c}_{1}\right)=\frac{16 G^{2} M^{2}}{9} \frac{1}{s^{4}}\left[\frac{11}{32}+\frac{5}{16} \cos ^{2} \theta+\frac{27}{32} \cos ^{4} \theta\right]
\end{aligned}
$$


Equation (44) is a second order inhomogeneous linear differential equation, whose solution is a superposition of a special solution and a solution of the corresponding homogeneous equation. In order to derive the contribution to the solution from the inhomogeneous terms $f_{i}, i=1,2,3$ we make the ansatz $\phi_{i}=\frac{A_{i}+B_{i} \cos ^{2} \theta+C_{i} \cos ^{4} \theta}{s^{2}}, i=1,2,3$ with $A_{i}, B_{i}, C_{i}$ constants. Then by demanding that

$$
\left[\frac{1}{s^{3}} \frac{\partial}{\partial s}\left(s^{3} \frac{\partial}{\partial s}\right)+\frac{1}{s^{2} \sin ^{2} \theta} \frac{\partial}{\partial \theta}\left(\sin ^{2} \theta \frac{\partial}{\partial \theta}\right)-\frac{3}{s} \frac{\partial}{\partial s}+\frac{3}{s^{2}} \tan \theta \frac{\partial}{\partial \theta}\right] \phi_{i}(s, \theta)=f_{i}, \quad i=1,2,3
$$

we calculate the coefficients $A_{i}, B_{i}, C_{i}$ and thus determine the part of the solution which corresponds to the inhomogeneous terms

$$
\begin{aligned}
& \phi_{1}=-\frac{G^{2} M^{2}}{s^{2}}\left(\frac{8}{9}+\frac{4}{9} \cos ^{2} \theta+\frac{8}{9} \cos ^{4} \theta\right), \quad \phi_{2}=-\frac{40}{9} \frac{G^{2} M^{2}}{s^{2}} \cos ^{4} \theta \\
& \phi_{3}=\frac{G^{2} M^{2}}{s^{2}}\left(\frac{7}{36}+\frac{5}{36} \cos ^{2} \theta-\frac{1}{4} \cos ^{4} \theta\right) .
\end{aligned}
$$

Thus, a special solution of the equation takes the form

$$
\phi=\phi_{1}+\phi_{2}+\phi_{3}=\frac{G^{2} M^{2}}{s^{2}}\left(-\frac{25}{36}-\frac{11}{36} \cos ^{2} \theta-\frac{67}{12} \cos ^{4} \theta\right) .
$$

The most general form of the solution to the homogeneous equation, which is consistent with the large $\kappa s$ approximation can be put in the form

$$
\frac{1}{s^{2}}\left[F_{0}(\theta)+F_{1}(\theta) \ln \kappa s+\ldots+F_{m}(\theta) \ln ^{m} \kappa s\right] .
$$

As is shown in the appendix, the ordinary differential equation satisfied by $F_{m}(\theta)$ can be converted into a hypergeometric equation with the two linearly independent solutions

$$
w_{1}(\theta)=\cos ^{3} \theta \cot \theta
$$

and

$$
w_{2}(\theta)=-\frac{1}{2}-\frac{3}{4} \cos ^{2} \theta+\frac{3}{8} \cos ^{3} \theta \cot \theta \ln \frac{1-\sin \theta}{1+\sin \theta} .
$$

$w_{1}$ is singular at $\theta=0\left(A d S_{5}\right.$ horizon $)$ and it should not be included while $w_{2}$ is finite at $\theta=\frac{\pi}{2}$, its inclusion though would produce on the brane terms of the form $\frac{1}{r^{2}} \ln ^{m} \kappa r$, in disagreement with the standard Schwarzschild metric. Thus we expect $F_{m}(\theta)=0$. Similarly we expect that $F_{m-1}(\theta), \ldots, F_{1}(\theta)$ are equal to zero and we are left with $a_{1}=$ $\phi+\eta w_{2}$, where $\eta$ is a constant to be determined.

Similarly to the solution of the linearized equations the physical solution to second order will be given by

$$
a_{1}^{P}=a_{1}+\delta a_{1}, \quad b_{1}^{P}=b_{1}+\delta b_{1}, \quad c_{1}^{P}=c_{1}+\delta c_{1} .
$$


The parameters of transformation that generate the residual coordinate transformations $v_{1}, u_{1}$ obey the constraints (14) to second order

$$
\begin{aligned}
2 \dot{v}_{1} & =-e^{-2 \kappa y} \dot{u}_{0}^{2}=-e^{2 \kappa y} v_{0}^{\prime 2} \\
v_{1}^{\prime}+e^{-2 \kappa y} \dot{u}_{1} & =-e^{-2 \kappa y}\left(b_{0}-2 \kappa v_{0}\right) \dot{u}_{0}-e^{-2 \kappa y} \dot{u}_{0} u_{0}^{\prime}
\end{aligned}
$$

where we have used equation (32), while the components of the metric transform to second order as

$$
\begin{aligned}
& \delta a_{1}=a_{0}^{\prime} u_{0}+\dot{a}_{0} v_{0}-2 \kappa v_{1} \\
& \delta b_{1}=b_{0}^{\prime} u_{0}+\dot{b}_{0} v_{0}-2 \kappa v_{1}+2 u_{1}^{\prime}-u_{0}^{\prime 2}+e^{2 \kappa y} v_{0}^{\prime 2} \\
& \delta c_{1}=c_{0}^{\prime} u_{0}+\dot{c}_{0} v_{0}-2 \kappa v_{1}+2 \frac{u_{1}}{r}-\frac{u_{0}^{2}}{r^{2}}
\end{aligned}
$$

and

$$
\delta h_{1} \equiv \delta a_{1}+\delta b_{1}+2 \delta c_{1}=-8 \kappa v_{1}+2 u_{1}^{\prime}+\frac{4}{r} u_{1}-u_{0}^{\prime 2}+e^{2 \kappa y} v_{0}^{\prime 2}-\frac{2 u_{0}^{2}}{r^{2}} .
$$

The constraints (51) yield the following expressions for $v_{1}$ and $\dot{u}_{1}$,

$$
\begin{aligned}
& \dot{v}_{1}=-\frac{1}{2 \kappa} e^{2 \kappa y} v_{0}^{\prime 2}+\lambda(r)=-e^{2 \kappa y} \frac{G^{2} M^{2}}{36 \kappa^{3} r^{4}}+\lambda(r) \\
& \dot{u}_{1}=e^{2 \kappa y}\left(b_{0}-2 \kappa v_{0}+u_{0}^{\prime}\right) v_{0}^{\prime}-e^{2 \kappa y} v_{1}^{\prime}
\end{aligned}
$$

where $\lambda(r)$ is an arbitrary fucntion of $r$ which can be determined by enforcing the brane bending condition-Neumann boundary condition to second order: more specifically,

$$
\left.\left(\dot{h}_{1}+\delta \dot{h}_{1}\right)\right|_{y=0}=2\left(-\lambda^{\prime \prime}-\frac{2}{r} \lambda^{\prime}-\frac{17 G^{2} M^{2}}{36 \kappa r^{4}}\right)=0
$$

We find that $\lambda(r)=-\frac{17 G^{2} M^{2}}{72 \kappa r^{2}}$ and consequently that

$$
v_{1}(r, y)=-e^{2 \kappa y} \frac{G^{2} M^{2}}{36 \kappa^{3} r^{4}}-\frac{17 G^{2} M^{2}}{72 \kappa r^{2}}
$$

A comment regarding the way Neumann boundary condition is met appears necessary at this point. Unlike the linearized solutions, which we have the exact expressions at our disposal, here only the approximate expressions at large $\kappa s$ are available. So we can not satisfy the Neumann boundary condition perfectly. On the other hand, it is easy to show that for large $\kappa r$ on the brane, $\dot{a}_{1}, \dot{b}_{1}$ and $\dot{c}_{1}$ are dominated by terms of order $\frac{G^{2} M^{2}}{\kappa r^{4}}$. It is this order that has to be cancelled by the gauge transformation in conformity to the Neumann boundary condition. In another word, $\dot{a}^{P}, \dot{b}^{P}$ and $\dot{c}^{P}$ should be of higher order than $\frac{G^{2} M^{2}}{\kappa r^{4}}$ at $y=0$ and should vanish exactly when higher powers of $1 / \kappa s$ are restored in the expressions of $a^{P}, b^{P}$ and $c^{P}$. 
Using equations (50), (52) and (56) we derive the following expression for $a_{1}^{P}$

$$
\begin{aligned}
a_{1}^{P}=-\frac{G^{2} M^{2}}{s^{2}} & {\left[\frac{25}{36}+\frac{16}{9} \csc \theta-\frac{17}{36} \csc ^{2} \theta+\left(\frac{11}{36}+\frac{4}{3} \csc \theta-\frac{1}{18} \csc ^{4} \theta\right) \cos ^{2} \theta\right.} \\
& \left.+\left(\frac{67}{12}-\frac{2}{3} \csc \theta\right) \cos ^{4} \theta+\eta w_{2}(\theta)\right]
\end{aligned}
$$

It is straightforward to verify that $a_{1}^{P}$ satisfies Neumann boundary condition $\dot{a}_{1}^{P}(r, 0)=0$ only if $\eta=0$. On the physical brane and for $\kappa r>>1$, the time component of the metric to second order reads

$$
-g_{00}=e^{a_{0}^{P}+a_{1}^{P}}=1-\frac{2 G M}{r}+O\left(\frac{G^{3} M^{3}}{r^{3}}\right)
$$

which is consistent with the General Relativity prediction $\gamma=0$.

For completeness we will determine the expression for $b_{1}$ and $c_{1}$ on the brane and for $\kappa r>>1$. Using the fact that $h_{1}=a_{1}+b_{1}+2 c_{1}$, the second of equations (40) takes the form

$$
\dot{b}_{1}^{\prime}+\frac{3}{r} \dot{b}_{1}=\dot{h}_{1}^{\prime}+\frac{1}{r} \dot{h}_{1}-\frac{1}{r} \dot{a}_{1}+\frac{1}{2}\left(a_{0}{ }^{\prime} \dot{a}_{0}+b_{0}{ }^{\prime} \dot{b}_{0}+2 c_{0}{ }^{\prime} \dot{c}_{0}\right)
$$

Furthermore, if we introduce $\beta=\dot{b}_{1}-\dot{h}_{1}$, equation (59) takes the simple form

$$
\beta^{\prime}+\frac{3}{r} \beta=f_{1}+f_{2}
$$

where $f_{1}=-\frac{2}{r} \dot{h}_{1}-\frac{1}{r} \dot{a}_{1}$ and $f_{2}=\frac{1}{2}\left(a_{0}{ }^{\prime} \dot{a}_{0}+b_{0}{ }^{\prime} \dot{b}_{0}+2 c_{0}{ }^{\prime} \dot{c}_{0}\right)$ Using now the new set of coordinates $s, \theta$ and integrating (60), we find that

$$
\beta=\frac{\kappa G^{2} M^{2}}{s^{2}}\left(\frac{14}{9}-\frac{56}{9} \cos ^{2} \theta-\frac{3}{2} \cos ^{4} \theta\right) \cos ^{2} \theta
$$

Inserting this expression into $\dot{b}_{1}=\beta+\dot{h}_{1}$ and integrating, we derive

$$
b_{1}=\frac{1}{3} \frac{G^{2} M^{2}}{s^{2}}+\frac{17}{12} \frac{G^{2} M^{2}}{s^{2}} \cos ^{2} \theta
$$

We still need to calculate the contribution from the residual coordinate transformations generated by $v_{1}, u_{1}$. According to equation (51), $b_{1}$ transforms as follows

$$
b_{1} \mapsto b_{1}+b_{0}^{\prime} u_{0}+\dot{b}_{0} v_{0}-2 \kappa v_{1}+2 u_{1}^{\prime}-u_{0}^{\prime 2}+e^{2 \kappa y} v_{0}^{\prime 2}
$$

with $v_{1}$ and $u_{1}$ given by equations (54). If we integrate the second of these equations, we derive an expression for $u_{1}$

$$
u_{1}=\frac{4}{9} \frac{G^{2} M^{2}}{\sin ^{2} \theta}-\frac{1}{8} \frac{G^{2} M^{2}}{s} \frac{\cot ^{2} \theta}{\sin \theta}+\psi
$$


where $\psi=\psi(r)$ is an arbitrary function of $r$. Combining equation (61) and the expression for $\delta b_{1}$, we find $b_{1}^{P}$. The Neumann bondary condition is satisfied for arbitrary $\psi$. Imposing though the requirement that $b_{1}^{P}$ reproduces the standard Schwarzschild metric for large $r$ determines $\psi$

$$
\psi(r)=-\frac{43}{72} \frac{G^{2} M^{2}}{r}
$$

Combining relations (62), (63), (54), and (65), we find

$$
\begin{aligned}
b_{1}^{P}=\frac{G^{2} M^{2}}{s^{2}} & {\left[\frac{1}{3}+\frac{8}{9} \sin \theta+\frac{8}{9} \csc \theta+\frac{5}{3} \csc ^{2} \theta-\frac{16}{9} \csc ^{3} \theta\right.} \\
& \left.+\left(\frac{17}{12}+\frac{2}{3} \csc \theta+\frac{11}{12} \csc ^{4} \theta\right) \cos ^{2} \theta-\frac{1}{9} \csc ^{6} \theta \cos ^{4} \theta\right]
\end{aligned}
$$

It follows from the definition of $h_{1}^{P}$ as well as the expressions of $a_{1}^{P}$ and $b_{1}^{P}$, that

$$
\begin{aligned}
c_{1}^{P}=\frac{G^{2} M^{2}}{s^{2}} & {\left[-\frac{1}{6}+\frac{4}{9} \sin \theta-\frac{7}{6} \csc ^{2} \theta+\frac{8}{9} \csc ^{3} \theta+\left(-\frac{3}{4}+\frac{4}{3} \sin \theta-\frac{1}{9} \csc \theta\right.\right.} \\
& \left.\left.-\frac{5}{12} \csc ^{4} \theta\right) \cos ^{2} \theta+\left(\frac{8}{3}+\csc \theta-\frac{1}{36} \csc ^{6} \theta\right) \cos ^{4} \theta\right]
\end{aligned}
$$

What is remarkable here is that without any further degrees of freedom, we have already

$$
c_{1}^{P}=O\left(\frac{G^{2} M^{2}}{\kappa^{2} r^{3}}\right) \simeq 0
$$

for $\cos \theta=\frac{1}{\kappa r}$ and $r>>1$. Thus, we have recovered the standard Schwarzschild metric at large distances on the physical brane to the first order of nonlinearity

$$
d s^{2} \simeq-\left(1-\frac{2 G M}{r}+\cdots\right) d t^{2}+\left(1+\frac{2 G M}{r}+\frac{4 G^{2} M^{2}}{r^{2}}+\cdots\right) d r^{2}+r^{2} d \Omega^{2}
$$

where the dots indicate terms of order $O\left(\frac{G^{3} M^{3}}{r^{3}}\right)$ and higher.

\section{Conclusions.}

In this section we will recapitulate what we have done in this paper. We considered the most general ansatz for a static, axially symmetric metric in $D=4+1$ dimensions in the Randall-Sundrum framework and derived the Einstein equations. We subsequently solved the equations to first order in the gravitational coupling and found an exact, singularity free solution of the linearized equations. This solution describes the gravitational field of a spherically symmetric mass distribution confined on the physical brane. At distances far away from the material source and on the physical brane this solution coincides with the four dimensional Schwarzschild metric. Thus we confirmed that all tests of Linearized 
General Relativity are satisfied. Furthermore, we proceeded to examine the solution of the Einstein equations to the second order in the gravitational coupling $G M$. We derived an approximate expression for the gravitational field which is valid to second order in the gravitational coupling in a region far away from the source. When this expression is confined on the physical brane it coincides again with the standard Scwarzschild form to second order and thus reproduces the correct formula for the precession angle of Mercury. These results reinenforce the belief that the Randall-Sundrum scenario reproduces General Relativity on the physical brane to all orders in the gravitational coupling. We hope to report soon our results towards this direction.

\section{Acknowledgments.}

We would like to thank N. Khuri, J. T. Liu, Y. Pang and M. Porrati for useful discussions. This work was supported in part by the Department of Energy under Contract Number DE-FG02-91ER40651-TASK B.

\section{Appendix}

The homogeneous equation (44),

$$
\left[\frac{1}{s^{3}} \frac{\partial}{\partial s}\left(s^{3} \frac{\partial}{\partial s}\right)+\frac{1}{s^{2} \sin ^{2} \theta} \frac{\partial}{\partial \theta}\left(\sin ^{2} \theta \frac{\partial}{\partial \theta}\right)-\frac{3}{s} \frac{\partial}{\partial s}+\frac{3}{s^{2}} \tan \theta \frac{\partial}{\partial \theta}\right] \mathcal{F}(s, \theta)=0
$$

is a Quasi-Laplace since the first two terms inside the bracket is the four-dimensional Laplacian acting on an axially symmetric function. Inspired by the large $s$ expansion in (31), we look for the solution of the form

$$
\frac{1}{s^{n}} \sum_{l=0}^{m} F_{l}(\theta) \ln ^{l} \kappa s
$$

with $n$ a positive integer. Substituting it into (70), we obtain a system of $m$ ordinary differential equations for $F_{l}$ 's

$$
\left[\frac{1}{\sin ^{2} \theta} \frac{\partial}{\partial \theta}\left(\sin ^{2} \theta \frac{\partial}{\partial \theta}\right)+3 \tan \theta \frac{\partial}{\partial \theta}+n(n+1)\right] F_{l}=(l+1)\left[(2 n+1) F_{l+1}-(l+2) F_{l+2}\right]
$$

If we further introduce a new parameter $\varrho=\cos ^{2} \theta$, the differential equations (72) are transformed into

$$
\left[\varrho(1-\varrho) \frac{d^{2}}{d \varrho^{2}}+\left(-1-\frac{1}{2} \varrho\right) \frac{d}{d \varrho}+\frac{1}{4} n(n+1)\right] F_{l}=\frac{1}{4}(l+1)\left[(2 n+1) F_{l+1}-(l+2) F_{l+2}\right]
$$

Since $F_{m+1}=F_{m+2}=0$, the equation for $F_{m} \equiv F$ is a hypergeometric equation

$$
\varrho(1-\varrho) \frac{d^{2} F}{d \varrho^{2}}+(\gamma-(\alpha+\beta+1) \varrho) \frac{d F}{d \varrho}-\alpha \beta F=0
$$


with $\alpha=\frac{n}{2}, \beta=-\frac{1}{2}(n+1)$ and $\gamma=-1$. The other equations are of the same hypergeometric type but with inhomogeneous terms. The set of equations can be solved successively for $F_{l}$ in a descending order of the subscript $l$. For odd $n, \beta$ is a negative integer and there is a polynomial solution. The companion solution, however, is nonanalytic at $\varrho=0$ or $\varrho=1$ or both. The terms of the large $s$ expansion (31) contains odd powers of $s$ and the power of the logarithm $\ln \kappa s$ does appear in association with the polynomial solution. For even $n$, the solution can be written as

$$
F=\frac{\bar{F}}{\sqrt{1-\varrho}}
$$

with $\bar{F}$ satisfying a different hypergeometric equation with $\alpha=\frac{1}{2}(n-1), \beta=-\frac{n}{2}-1$ and $\gamma=-1$. This new hypergeometric equation possesses a polynomial solution. But the square root in (75) does not make it an analytic solution for $F$. The case with $n=2$ is of special interest. In this case one of the solutions is

$$
w_{1}(\varrho)=\frac{\varrho^{2}}{\sqrt{1-\varrho}}=\cos ^{3} \theta \cot \theta
$$

Given the solution $w_{1}$ we can derive the second solution by means of the standard formula,

$w_{2}(\varrho)=w_{1} \int^{\varrho} d \xi w_{1}^{-2} e^{-\int^{\xi} d \eta p(\eta)}$ where $p(\eta)=-\frac{1+\frac{1}{2} \eta}{\eta(1-\eta)}$. Subsequently we find that the second solution to the homogeneous differential equation is given by

$$
\begin{aligned}
w_{2}(\varrho) & =-\frac{1}{2}-\frac{3}{4} \varrho+\frac{3}{8} \frac{\varrho^{2}}{\sqrt{1-\varrho}} \ln \frac{1-\sqrt{1-\varrho}}{1+\sqrt{1-\varrho}} \\
& =-\frac{1}{2}-\frac{3}{4} \cos ^{2} \theta+\frac{3}{8} \cos ^{3} \theta \cot \theta \ln \frac{1-\sin \theta}{1+\sin \theta}
\end{aligned}
$$

\section{References.}

[1] L. Randall and R. Sundrum, Phys. Rev. Lett. 83 (1999) 4690

[2] K. Akama, Pregeometry, in Gauge Theory and Gravitation, edited by K. Kikkawa, N. Nakanishi and H. Nariai, Springer Verlag, Berlin (1983) 267, V. A. Rubakov, and M. E. Shaposhnikov, Phys. Lett. 125B (1983) 136, M. Visser, Phys. Lett. 159B (1985) 22, N. Arkani-Hamed, S. Dimopoulos and G. Dvali, Phys. Lett. 429B (1998) 263, I. Antoniadis, N. Arkani-Hamed, S. Dimopoulos and G. Dvali, Phys. Lett. 436B (1998) 257.

[3] R. Altendorfer, J. Bagger and D. Nemeschansky, "Supersymmetric Randall-Sundrum Scenario", hep-th/0003117.

[4] C. Charmousis, R. Gregory and V. A. Rubakov, "Wave function of the radion in a brane world", hep-th/9912160; R. Gregory, V. A. Rubakov, and S. M. Sibiryakov, Phys. Rev. Lett. 84 (2000) 5928, hep-th/0002072; I. Kogan, S. Mouslopoulos, A. 
Papazoglou, G. Ross and J. Santiago, "A three-brane universe: New phenomenology for the new millenium?", hep-th/9912552.

[5] C. Csaki, J. Erlich, and T. Hollowood, Phys. Lett. 481B (2000) 107, hep-th/0003020; G. Dvali, G. Gabadadze, and M. Porrati, " 4 D Gravity on a Brane in 5D Minkowski Space", hep-th/0005016; Phys. Lett. 484B (2000) 112, hep-th/0002190; R. Gregory, V. A. Rubakov, and S. M. Sibiryakov, "Gravity and Antigravity in a brane world with metastable gravitons", hep-th/0003045; C. Csaki, J. Erlich, T. Hollowood, and J. Terning "Holographic RG and Cosmology in Theories with Quasi-Localized Gravity", hep-th/0003076.

[6] A. Chamblin, S. W. Hawking and H. S. Reall, Phys. Rev. D61 (2000) 065007.

[7] A. Chamblin and G. W. Gibbons, Phys. Rev. Lett. 84 (2000) 1090.

[8] W. Israel, Nuovo Cimento B44 (1966) 1.

[9] I. Y. Arefe'va, M. Ivanov, W. Muck, K. S. Viswanathan, and I. V. Volovich, "Consistent linearized gravity in brane backgrounds", hep-th/0004114; M. Ivanov and I. V. Volovich, Metric fluctuations in brane worlds, hep-th/9912242; H. Collins and B. Holdom, Linearized Gravity about a Brane, hep-th/0006158.

[10] W. Muck, K. S. Viswanathan and I. V. Volovich, "Geodesics and Newton's Law in Brane Backgrounds", hep-th/0002132.

[11] J. Garriga and T. Tanaka, Phys. Rev. Lett. 84 (2000) 2778

[12] M. J. Duff and J. T. Liu, "On the Equivalence of the Maldacena and Randall-Sundrum Pictures", hep-th/0003237; R. Dick and D. Mikulovicz, Phys. Lett. 476B (2000) 363, hep-th/0001013.

[13] S. Weinberg, Gravitation and Cosmology, Chapter 8, John Wiley and Sons, Inc. (1972).

[14] Y. Myung, G. Kang, and H. Lee, Phys. Lett. 478B (2000) 294, hep-th/0001107.

[15] S. Giddings, E. Katz and L. Randall, JHEP 0003 (2000) 023. 